\title{
Giant Cell Myocarditis Following COVID-19 Successfully Treated by Immunosuppressive Therapy
}

\author{
Afsaneh Amiri ${ }^{1}$, Golnaz Houshmand ${ }^{2}$, Sepideh Taghavi ${ }^{3}$, Monireh Kamali ${ }^{4}$, and Nasim \\ Naderi $^{5}$ \\ ${ }^{1}$ Rajaie cardiovasular medical and research center,Iran University of medical sciences \\ ${ }^{2}$ Rajaie cardiovascular, medical and research center,Iran University of medical sciences \\ ${ }^{3}$ Rajaie cardiovascular medical and research center,Iran University of medical sciences \\ ${ }^{4}$ Iran University of Medical Sciences \\ ${ }^{5}$ Rajaie Cardiovascular Medical and Research Center,Iran university of medical sciences
}

December 22, 2021

\begin{abstract}
In this case report, we present a 46-year-old lady who has developed a rapidly progressive heart failure after an episode of COVID-19. The pathologic examination of her endomyocardial biopsy specimens was compatible with GCM and she was successfully treated with a combined immunosuppressive therapy regimen.
\end{abstract}

\section{Introduction:}

Giant cell myocarditis (GCM) is a rare and rapidly progressing form of myocarditis of autoimmune origin, histologically characterized by the presence of giant multinucleated cells along with extensive inflammatory infiltrates and is often associated with autoimmune disorder. ${ }^{1-4}$

COVID-19 has been considered as an established etiology of acute myocarditis. ${ }^{5-8}$ However, there is only one case report other than the present case regarding the development of GCM following COVID-19. ${ }^{9}$ Here, we are going to report a case of GCM following COVID-19 infection in a lady with a history of lichen plan dermatitis and hypothyroidism.

\section{Case Presentation:}

A 46-year-old female referred to heart failure clinic for further evaluations of her new onset heart failure. In her first visit on January 2021, she complained of a dyspnea of exertion, New York Heart Association functional class of II (NYHA-FC) and fatigue.

Her past medical history was unremarkable in terms of cardiovascular problems before her recent episode of acute heart failure. She had a history of a controlled lichen plan dermatitis (LP) treated by immunosuppressive therapy several years ago. She also had a history of antithyroid peroxidase antibody (TPO) positive hypothyroidism that was managed with levothyroxine.

A month before the first visit in our clinic (on December 2020), she had developed symptoms of COVID-19 such as fever, sore throat and malaise. The real time polymerase chain reaction (RT-PCR) test for the novel 
corona virus infection was positive but the clinical course of her COVID-19 infection was uneventful without any decrease in arterial $\mathrm{O} 2$ saturation or pulmonary involvement and the disease was subsided using the recommended medications for mild COVID-19.

About three weeks later (a week after the remission of the COVID-19 infection), she was admitted due to a severe chest discomfort and shortness of breath. Her electrocardiogram (ECG) was highly suggestive of acute anterior myocardial infarction (ST segment elevation in V2-V6 leads), according to her hospital discharge note. Considering the ECG changes and an elevated cardiac Troponin-I level, she underwent emergent coronary angiography, which revealed normal epicardial coronary arteries. Her echocardiogram reported a normal left ventricular (LV) size, with apical hypokinesia and an ejection fraction (EF) of 35-40\%, normal right ventricular (RV) size and function, a mild mitral valve regurgitation (MR), a mild tricuspid valve regurgitation (TR), no pulmonary hypertension and no pericardial effusion.

With an impression of acute myocarditis, she underwent a cardiac magnetic resonance imaging (CMR), and based on the results (Figure 1), she was referred to our heart failure clinic for further evaluations.

\section{CMR report:}

The CMR report revealed mildly enlarged LV size without LV hypertrophy with moderately reduced LV systolic function $\mathrm{LVEF}=35 \%$ and thin and aneurysmal LV apex -Normal RV size with mildly reduced RV function $(\mathrm{RVEF}=42 \%)$.

LGE findings: In all inferolateral and septal segments, there was widespread patchy subepicardial to mid wall fibrosis, as well as sub endocardial fibrosis in the left ventricular apex.

Recent myocarditis possibly due to viral infection, as well as an autoimmune or sarcoidosis disease, should be evaluated, according to CMR tissue characterization criteria.

\section{(Figure 1)}

On the first visit in our clinic on January 2021, she complained of a dyspnea on exertion, NYHA-FC II and a mild fatigue. Her vital signs were stable with a blood pressure (BP) of $115 / 75 \mathrm{mmHg}$ a heart rate (HR) of 90 beats per minute (bpm), she did not have fever and systemic oxygen saturation was $96 \%$.

Her physical examination was unremarkable except for bi-basilar fine crackles. She did not have any skin lesions in terms of her LP. Her new echocardiogram revealed no new changes compared to her last exam that was mentioned earlier. On laboratory tests, there was no leukocytosis, hemoglobin was $12.1 \mathrm{~g} / \mathrm{dl}$, the renal and liver function tests were within normal limits and $\mathrm{N}$ terminal -pro natriuretic peptide (NT-pro BNP) was $885 \mathrm{pg} / \mathrm{ml}$. The thyroid function test was within normal limits with a higher than normal anti TPO.

The standard anti failure therapies was already started and the heart failure (HF) guideline directed medical therapies with lisinopril, bisoprolol, eplerenone, furosemide as well as levothyroxine were continued for her. A few days later, her HF symptoms were aggravated and she developed progressive dyspnea, orthopnea, paroxysmal nocturnal dyspnea, abdominal pain and nausea. She was admitted with the above mentioned symptoms and rapidly progressed to a pre-shock state. She had a BP of $85 / 60$, an HR of $120 \mathrm{bpm}$, a distended jugular vein, bi basilar lung crackles, hepatomegaly, mild ascites and $2+$ pretibial pitting edema. The creatinine was slightly increased $(1.7-1.8 \mathrm{mg} / \mathrm{dl})$. The alanine aminotransferase (ALT), aspartate aminotransferase (AST) and total bilirubin levels were $46 \mathrm{U} / \mathrm{L}, 40 \mathrm{U} / \mathrm{L}, 1.5 \mathrm{mg} / \mathrm{dl}$, respectively, NT-Pro BNP was more than $18000 \mathrm{pg} / \mathrm{ml}$ and cardiac troponin -I (CTnI) was elevated. Her ECG showed a sinus tachycardia with low voltage QRSs and Q wave in leads of I, III, aVF, V1-V6, ST segment elevations and T wave inversions/flattening in V1-V6. (Figure 2)

New echocardiography showed mild LV enlargement with severe systolic dysfunction, LVEF 15-20\%, global hypokinesia and apical akinesia mild RV enlargement with severe systolic dysfunction [Tricuspid annular plane systolic excursion( TAPSE) $=10 \mathrm{~mm}$, RV peak systolic myocardial velocity by tissue Doppler (RV $\mathrm{Sm})=6 \mathrm{~cm} / \mathrm{sec}$, moderate to severe mitral (MR) and tricuspid regurgitation(TR), a tricuspid regurgitation gradient (TRG) of $25 \mathrm{mmHg}$ and a plethoric inferior vena cava (IVC) with an IVC size of 23 millimeter. 
Considering her clinical condition, we had to start intravenous (IV) inotrope and diuretic. After starting intravenous (IV) inotrope (Milrinone) and furosemide infusion, she was scheduled for emergent endomyocardial biopsy (EMBX) due to deterioration in hemodynamic and clinical course. The right heart catheterization data at the time of EMBX has been shown in table 1;

Table 1: Hemodynamic findings of patient

\begin{tabular}{ll}
\hline Catheterization data & Value \\
\hline Cardiac output by Fick method & 2.4 Liters/minute \\
Cardiac index by Fick method & $1.4 \mathrm{Liters} /$ kilogram of body weight/minute \\
Mean central venous pressure & $16 \mathrm{mmHg}$ \\
Mean Right ventricular pressure & $32 / 10-20 \mathrm{mmHg}$ \\
Pulmonary arterial pressure & $32 / 16 \mathrm{mmHg}$ \\
Pulmonary capillary wedge pressure & $17 \mathrm{mmHg}$ \\
Systemic arterial pressure & $90 / 60 \mathrm{mmHg}$ \\
Mixed venous oxygen saturation & $48 \%$ \\
Systemic arterial saturation & $98 \%$ \\
\hline
\end{tabular}

Five pieces of myocardial tissue from right ventricular side of interventricular septum were obtained and sent for both pathological examination and tissue polymerase chain reaction (PCR) for all cardiotropic viruses including influenza type $\mathrm{A}$ and $\mathrm{B}$, adenovirus, enterovirus, parvo virus B-19, cytomegalovirus, Human herpesvirus 6 (HHV-6) as well as the novel coronal virus.

Tissue PCR for all cardiotropic pathogens and the novel corona were negative. Autoimmune and connective tissue disease serology was unremarkable.

The microscopic exam of tissue demonstrated the myocardial fibers with multiple foci of mixed infiltration of inflammatory cells including lymphocytes, eosinophils, macrophages and multinucleated giant cells associated by focal myocyte damage and areas of fibrosis mainly in subendocardial areas compatible with active myocarditis highly suggestive for GCM. (Figure 3)

Immediately after reporting the pathology examination, a combined immunosuppressive therapy with IV pulse of methylprednisolone (500 $\mathrm{mg}$ for 3 days) and intravenous immune globin ([IVIG ( $500 \mathrm{mg} / \mathrm{kg}$ for 3 days)] was prescribed for the patient. The IV pulse therapy with methylprednisolone was followed by $1 \mathrm{mg} / \mathrm{Kg}$ oral prednisolone which was tapered by $5 \mathrm{mg}$ daily. The tablet of mycophenolate mofetil $1000 \mathrm{mg}$, 2 times daily got also started for her. The oral tacrolimus, $1 \mathrm{mg}$ two times daily got also started for her and adjusted to achieve the goal for a 12 hours trough whole blood level of 10-15 ng/ml for her first 6 months.

On the third day of treatment, the patient's clinical condition and symptoms were remarkably improved and we could decrease and stop the IV inotrope and change the diuretic to oral doses. At the end of the week her echocardiographic findings were significantly improved, LVEF increased to about 30\%, TAPSE to $14 \mathrm{~mm}$ and RV sm to $8 \mathrm{~cm} / \mathrm{sec}$.

The hospital course was uneventful and she had no arrhythmias and/or hemodynamic deterioration. Reviewing the 48-hour ECG Holter monitoring showed no abnormalities in terms of arrhythmias and/or atrioventricular block and the patient was discharged after a week with a good clinical condition a normal kidney and liver function test, an NT-Pro BNP of $1580 \mathrm{pg} / \mathrm{ml}$ and a normal CTnI . A week later, the patient continued to be free of symptoms and her new echocardiography showed further improvement; mild LV enlargement with an $\mathrm{EF}$ to $40 \%$, the apical segment of LV was still severe hypokinetic. mild to moderate MR, near normal RV size and function with a TAPSE of:18 mm and RVsm of $11 \mathrm{~cm} / \mathrm{sec}$ and mild TR and IVC diameter of $17 \mathrm{~mm}$ with good respiratory collapse.

The prednisolone daily dose was tapered to $30 \mathrm{mg}, 2$ grams daily dose of mycophenolate mofetil and tacrolimus was continued for her. At the end of $6^{\text {th }}$ month of treatment, she had no clinical symptoms, 
the echocardiography demonstrated the same findings, so the dose of oral prednisolone was reduced to 10 $\mathrm{mg} /$ day. On the $3^{\text {rd }}$ and $6^{\text {th }}$ month of follow-up her NT pro BNP level was $19.5 \mathrm{pg} / \mathrm{ml}$ and $30 \mathrm{pg} / \mathrm{ml}$ respectively.

\section{Discussion:}

COVID-19 may present with cardiovascular manifestations and complications. Although, currently it has been considered as an established etiology of myocarditis, there is no clear recommendation and consensus regarding the best clinical approach and the management of myocarditis following COVID-19. ${ }^{6-8,10-13}$

Here, we reported, a case of GCM following COVID-19 infection. Recently, Blagova et al. have reported a cardiomyopathy that was rapidly progressed to a sudden cardiac death following COVID -19 in a patient with a history of chronic adrenal insufficiency and myocardial disease with a final diagnosis GCM. ${ }^{9}$ As far as we researched, our case seems to be the first case report of GCM following COVID-19 in a patient with a background of autoimmune disorder, without any cardiac problem who was successfully treated with immunosuppressive therapy.

The etiology of GCM is unknown; however, an association with autoimmune diseases has been suggested. Many GCM cases are patients with immunological based diseases, such as thymoma, myasthenia gravis, dermatomyositis, thyroiditis, and pernicious anemia..$^{1,3,4,14-16}$ This association has prompted an autoimmune hypothesis for the etiology of GCM. Our patient had a history of an autoimmune dermatologic and thyroid disease.

It has been suggested that, patients with chronic and long term lichen plan (LP) dermatitis particularly those with erosive LP may have arterial stiffness secondary to autoimmune endothelial dysfunction and should be followed up in terms of cardiovascular disorders. However, the combination of GCM with autoimmune dermatologic including lichen planus has not been described yet. ${ }^{17,18}$

Another issue which should be considered in patients with LP dermatitis is its differential diagnosis with sarcoidosis. In a case series, Goldberg et al, have suggested that the LP can induce epidermotropic multinucleated giant cell inflammatory response and sarcoidosis with systemic involvement should be considered and worked up in these patients. ${ }^{19}$

Cardiac sarcoidosis (CS) could be considered as differential diagnosis of our patient. However, although cardiac sarcoidosis and giant cell myocarditis can be pathologically similar and pathological differentiation between the two may be difficult, cardiac sarcoidosis is not known to cause fulminant myocarditis and typically has a chronic course. Although there are a few reports of fulminant cardiac sarcoidosis, the treatment response of our patient could be in favor of GCM than CS. ${ }^{15,20-23}$

Since the beginning of the COVID-19 disaster, physicians have encountered a variety of clinical manifestations and complications of this disease and it seems that we should expect a stranger behavior of this virus overtime. COVID-19 is associated with a hyperinflammation and activated humoral and cell-mediated immune response in human body, particularly in its severe forms. ${ }^{8,12}$

Although, the mechanisms of COVID-19-related myocardial injury are not completely understood, the role of severe inflammatory response with significant cytokines release, predominantly interleukin-6 (IL-6), has been recognized. Data from COVID-19 victims show mechanisms such as vasculitis, extended endotheliitis, lymphocyte infiltration, and direct injury of cardiomyocytes by binding spike protein of the SARS-CoV-2 to the membrane protein angiotensin-converting enzyme 2, which may be involved in acute cardiac injury and myocarditis following COVID-19. - $-12,24,25^{2}$

The presence of microvascular dysfunction accompanied with a myocardial inflammatory process may be an explanation for the sub-endocardial ischemic pattern noticed in cardiac magnetic resonance of patients with COVID-19 like what was seen in our patient and the case reported by Blagova et al. ${ }^{9}$

Although the clinical presentation of COVID-19 was mild, we believe that the autoimmune reactions following COVID-19 in our patient, who had a background of autoimmune abnormality, have caused GCM 
which was successfully controlled by immunosuppressive therapies. Choosing an optimal evidence-based immunosuppression regimen was challenging in this setting.

We opted to employ a combined immunosuppressive therapy utilizing IVIG, corticosteroid, tacrolimus and mycophenolate mofetil for our patient based on earlier reports concerning the treatment of GCM and our center's previous experience with the treatment of GCM and recent COVID-19 infection. ${ }^{1,2,5,13,14,16}$

However, several questions remained unanswered which will be clarified over time. First, as there are several reports of acute myocarditis following COVID-19 vaccination, which of the COVID-19 vaccines would be safe in patients who had myocarditis following COVID-19 infection, particularly those with inflammatory myocarditis such as GCM. ${ }^{26,27}$ Secondly, the necessity of further systemic work up including FDG-PET or CMR and/or repeated EMBx to exclude CS or for prognostication. Third, what would be the correct surveillance for electrophysiologic complications in this patient? Despite the fact that our patient never developed arrhythmias or atrioventricular block, her LVEF increased to $40 \%$ and up to now she remains asymptomatic, we advised her for ICD implantation for the primary prevention.

In conclusion, it seems that the development of myocarditis in COVID 19 would be independent of the severity of the illness. The current report supports the role of autoimmunity and immunosuppressive therapy in COVID-19 myocarditis including the inflammatory ones such as GCM. Further investigations are needed to determine the appropriate diagnostic and prognostic approach in patients with this type of myocarditis and necessity of surveillance regarding the myocardial inflammatory disorders in known cases of autoimmune diseases who have COVID-19 infection without cardiac injury.

\section{Conflict of interest:}

None

\section{Acknowledgments:}

We would like to thank you our colleagues Dr. Ali Hesami (Mostoufi Lab.) for prompt evaluation and report of endomyocardial biopsy specimen as well as Dr. Nick Austin for the language editing of the manuscript.

\section{Figure legends}

Figure 1 Fig1 A, B) Short tau inversion recovery (STIR) image showing high signal intensity in the septal and lateral wall

A: 


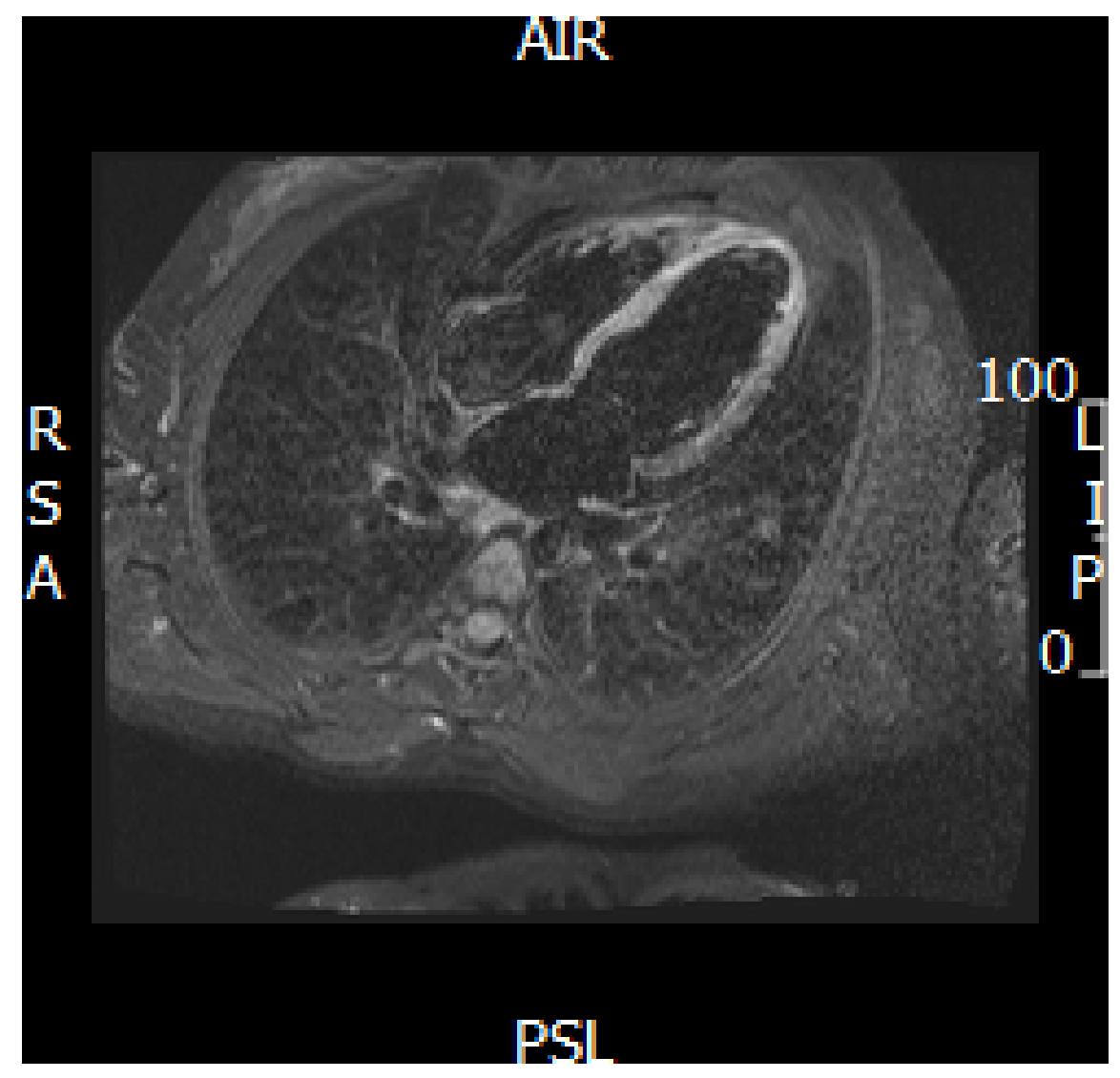

B: 


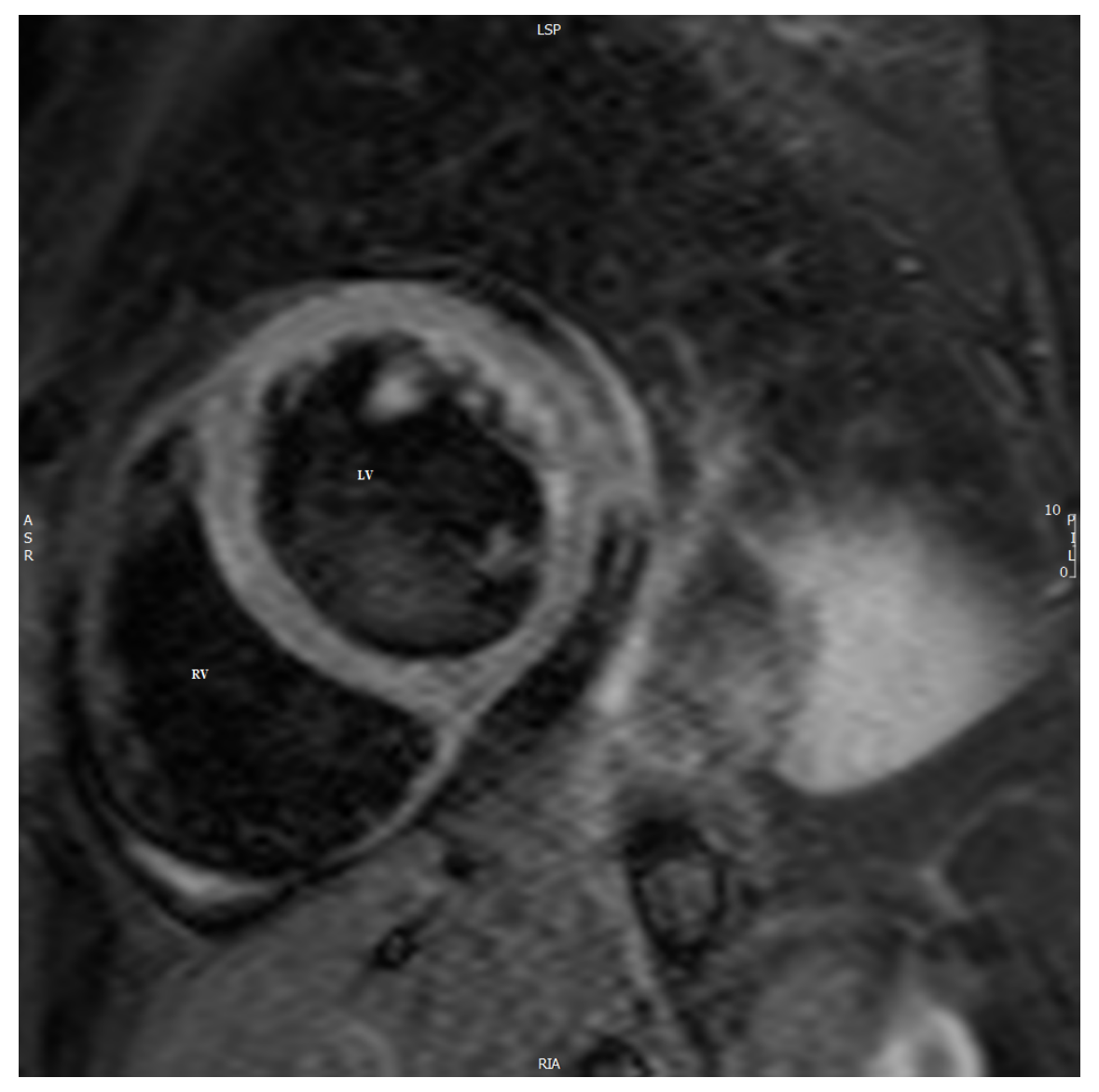

Fig 1 C,D) Late gadolinium enhancement images showing mixed pattern of myocardial injury including subendocardial and mid myocardial enhancement

C: 


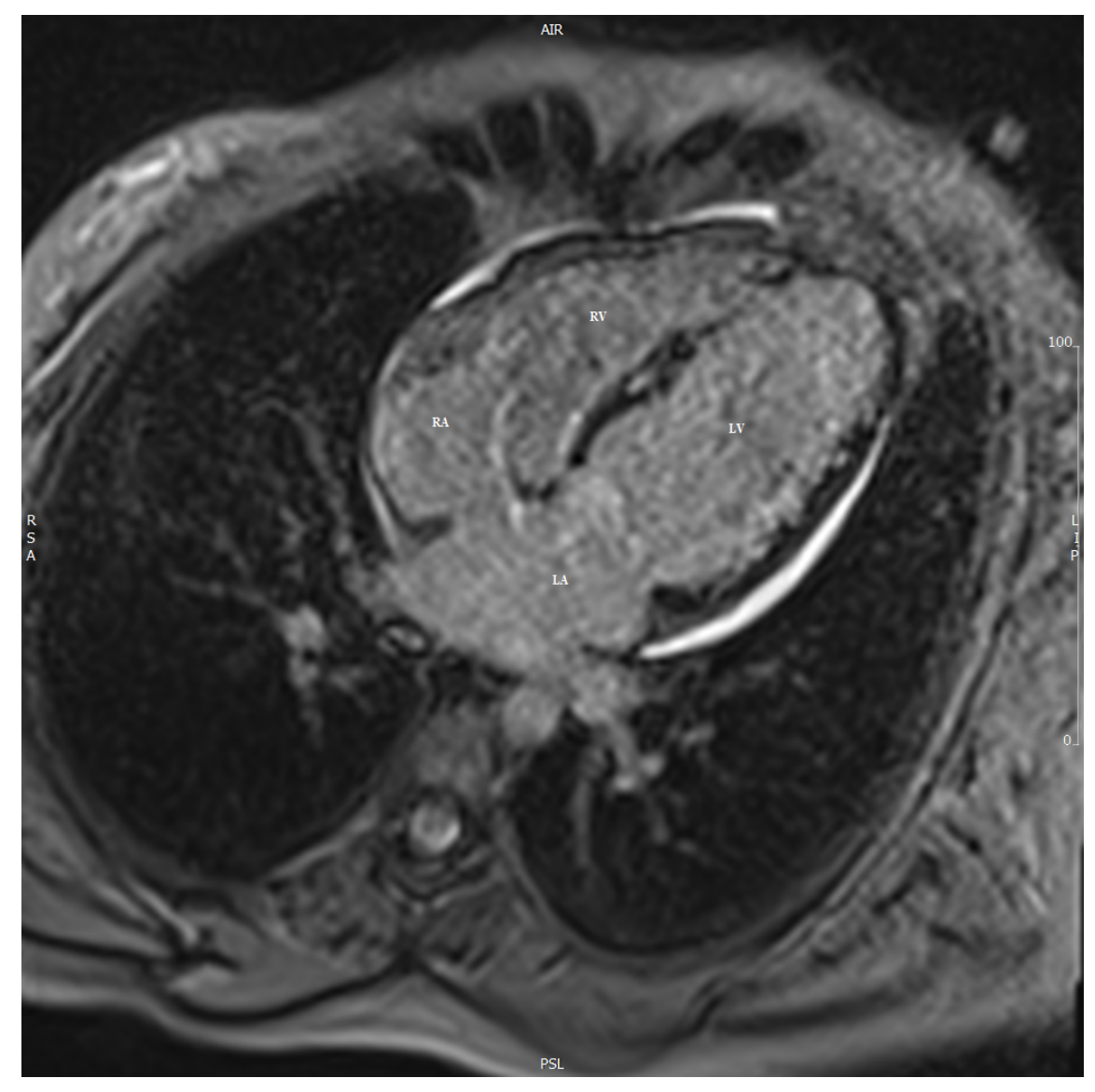

D: 


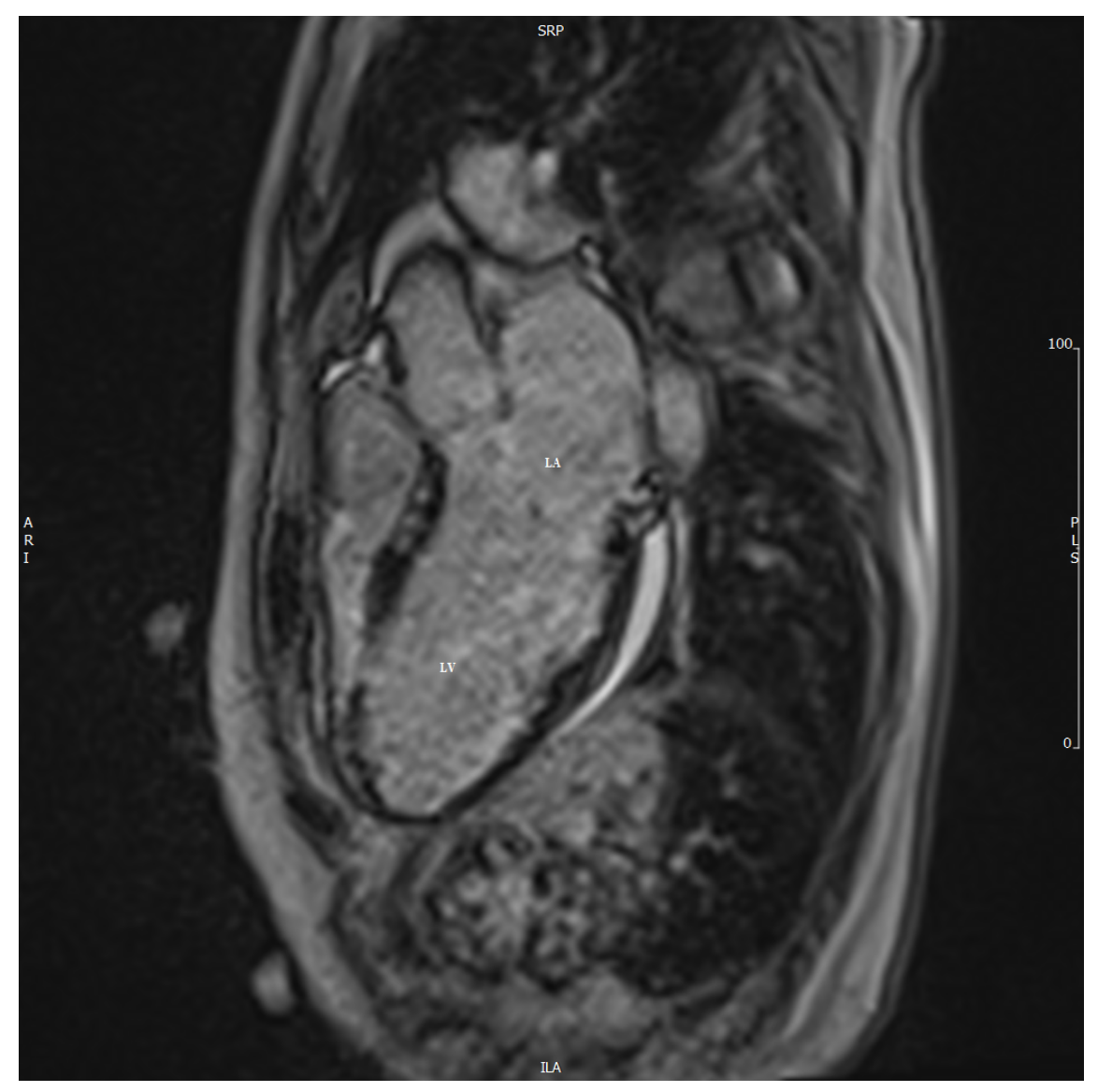

Figure 2: Electrocardiogram of patient at the time of admission.

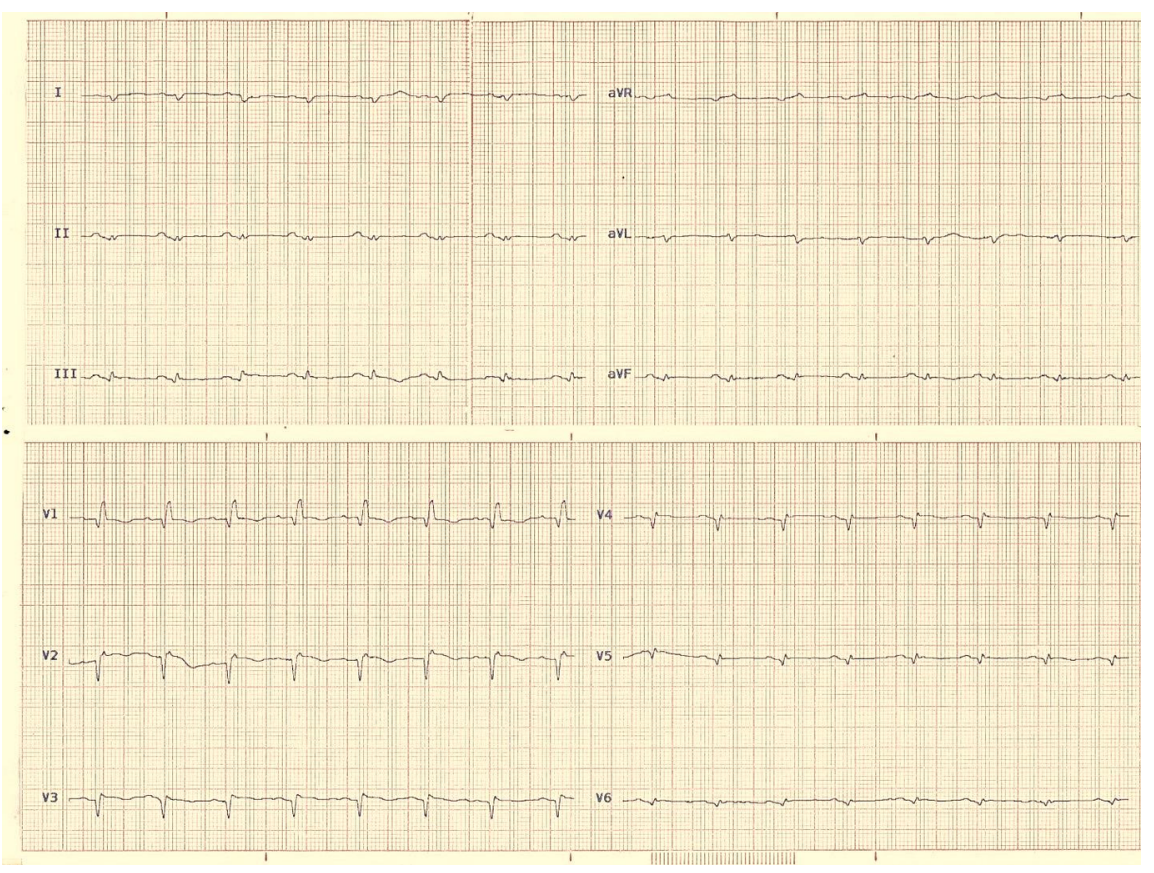


Figure 3 A-B: Microscopic examination of endomyocardial biopsy specimens shows the myocardial fibers with multiple foci of mixed infiltration of inflammatory cells including lymphocytes, eosinophils, macrophages and multinucleated giant cells (B-the blue arrows) associated by focal myocyte damage

A:

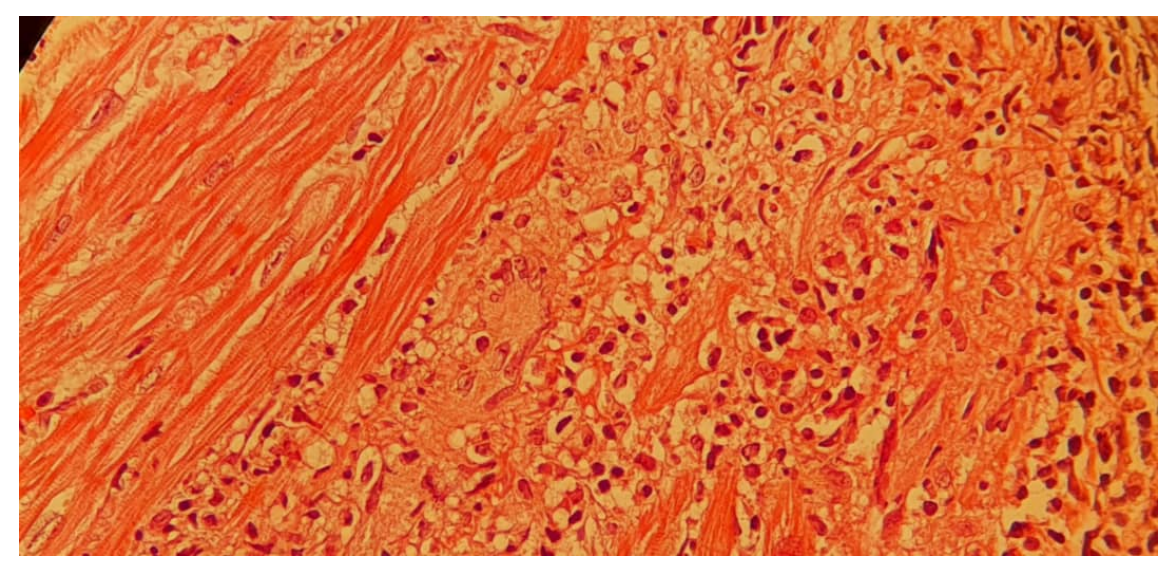

B:

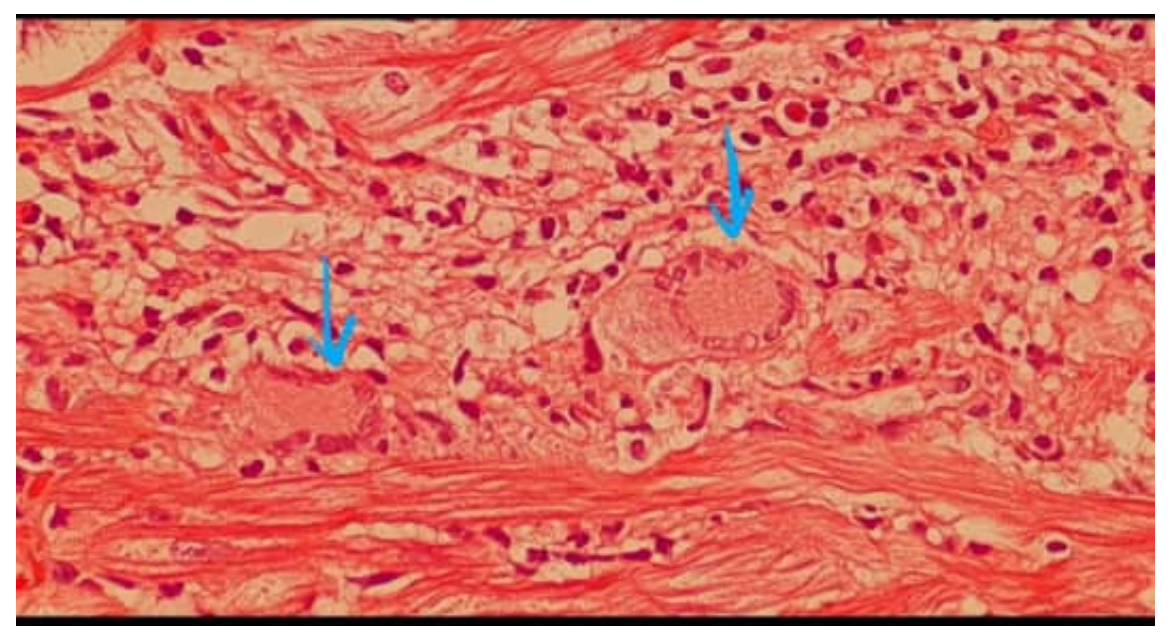

\section{References:}

1. Cooper Jr LT, Berry GJ, Shabetai R. Idiopathic giant-cell myocarditis-natural history and treatment. New England Journal of Medicine.1997;336(26):1860-1866.

2. Fallon JM, Parker AM, Dunn SP, Kennedy JL. A giant mystery in giant cell myocarditis: navigating diagnosis, immunosuppression, and mechanical circulatory support.ESC heart failure. 2020;7(1):316-320.

3. Meyer T, Grumbach IM, Kreuzer H, Morguet AJ. Giant cell myocarditis due to coxsackie B2 virus infection. Cardiology. 1997;88(3):296-299.

4. Xu J, Brooks EG. Giant cell myocarditis: a brief review. Archives of pathology $\& 3$ laboratory medicine. 2016;140(12):1429-1434.

5. Mele D, Flamigni F, Rapezzi C, Ferrari R. Myocarditis in COVID-19 patients: current problems. Internal and Emergency Medicine. 2021:1-7. 
6. Rathore SS, Rojas GA, Sondhi M, et al. Myocarditis associated with Covid-19 disease: A systematic review of published case reports and case series.International Journal of Clinical Practice. n/a(n/a):e14470.

7. Shi S, Qin M, Cai Y, et al. Characteristics and clinical significance of myocardial injury in patients with severe coronavirus disease 2019. European heart journal. 2020;41(22):2070-2079.

8. Yang F, Cao S, Liu X, Xiang Y, Zhao Q. Characteristics and clinical significance of myocardial injury in patients with severe coronavirus disease 2019. European Heart Journal. 2020;41:2070-2079.

9. Blagova O, Ainetdinova D, Sedov A, et al. Progressive chronic SARS-CoV-2-positive giant cell myoendocarditis with atrial standstill and sudden cardiac death.ESC Heart Failure. 2021.

10. Agdamag ACC, Edmiston JB, Charpentier V, et al. Update on COVID-19 myocarditis.Medicina. 2020;56(12):678.

11. Blauwet LA, Cooper LT. Myocarditis. Progress in cardiovascular diseases.2010;52(4):274-288.

12. Ramandi MMA, Yarmohammadi H, Beikmohammadi S, et al. Comparison of the cardiovascular presentations, complications and outcomes following different coronaviruses' infection: A systematic review. Journal of Cardiovascular and Thoracic Research. 2021;13(2):92-101.

13. Bang V, Ganatra S, Shah SP, et al. Management of Patients With Giant Cell Myocarditis. Journal of the American College of Cardiology.2021;77(8):1122-1134.

14. Cooper Jr LT, Hare JM, Tazelaar HD, et al. Usefulness of immunosuppression for giant cell myocarditis. The American journal of cardiology.2008;102(11):1535-1539.

15. Ekström K, Räisänen-Sokolowski A, Lehtonen J, Nordenswan HK, Mayranpaa MI, Kupari M. Idiopathic giant cell myocarditis or cardiac sarcoidosis? A retrospective audit of a nationwide case series. ESC heart failure. 2020;7(3):1362-1370.

16. Kandolin R, Lehtonen J, Salmenkivi K, Raisanen-Sokolowski A, Lommi J, Kupari M. Diagnosis, treatment, and outcome of giant-cell myocarditis in the era of combined immunosuppression. Circulation: Heart Failure. 2013;6(1):15-22.

17. Baykal Selcuk L, Sahin M, Arıca DA, Orem A, Karaca Ural Z, Yaylı S. Impairment of myocardial functions and arterial stiffness in patients with lichen planus,. Anais brasileiros de dermatologia. 2020;95:180186.

18. Gonzalez JG, Marcus MD, Santa Cruz DJ. Giant cell lichenoid dermatitis. Journal of the American Academy of Dermatology. 1986;15(1):87-92.

19. Goldberg LJ, Goldberg N, Abrahams I, Silvers DN, Szaniawski W, Halperin AJ. Giant cell lichenoid dermatitis: A possible manifestation of sarcoidosis. Journal of Cutaneous Pathology. 1994;21(1):47-51.

20. Birnie DH, Nair V, Veinot JP. Cardiac Sarcoidosis and Giant Cell Myocarditis: Actually, 2 Ends of the Same Disease? Journal of the American Heart Association. 2021;10(6):e020542.

21. Jiang GY, Cai Q, Grandin EW, Sabe MA. Fulminant cardiac sarcoidosis resembling giant cell myocarditis: a case report. European Heart Journal - Case Reports. 2021;5(3).

22. Nordenswan HK, Lehtonen J, Ekström K, et al. Manifestations and outcome of cardiac sarcoidosis and idiopathic giant cell myocarditis by 25-year nationwide cohorts. Journal of the American Heart Association. 2021;10(6):e019415.

23. Okura Y, Dec GW, Hare JM, et al. A clinical and histopathologic comparison of cardiac sarcoidosis and idiopathic giant cell myocarditis. Journal of the American College of Cardiology. 2003;41(2):322-329.

24. Schaller T, Hirschbuhl K, Burkhardt K, et al. Postmortem examination of patients with COVID19. Jama. 2020;323(24):2518-2520. 
25. Varga Z, Flammer AJ, Steiger P, et al. Endothelial cell infection and endotheliitis in COVID-19. The Lancet. 2020;395(10234):1417-1418.

26. Diaz GA, Parsons GT, Gering SK, Meier AR, Hutchinson IV, Robicsek A. Myocarditis and pericarditis after vaccination for COVID-19. JAMA. 2021.

27. Hudson B, Mantooth R, DeLaney M. Myocarditis and pericarditis after vaccination for COVID19. Journal of the American College of Emergency Physicians Open.2021;2(4).

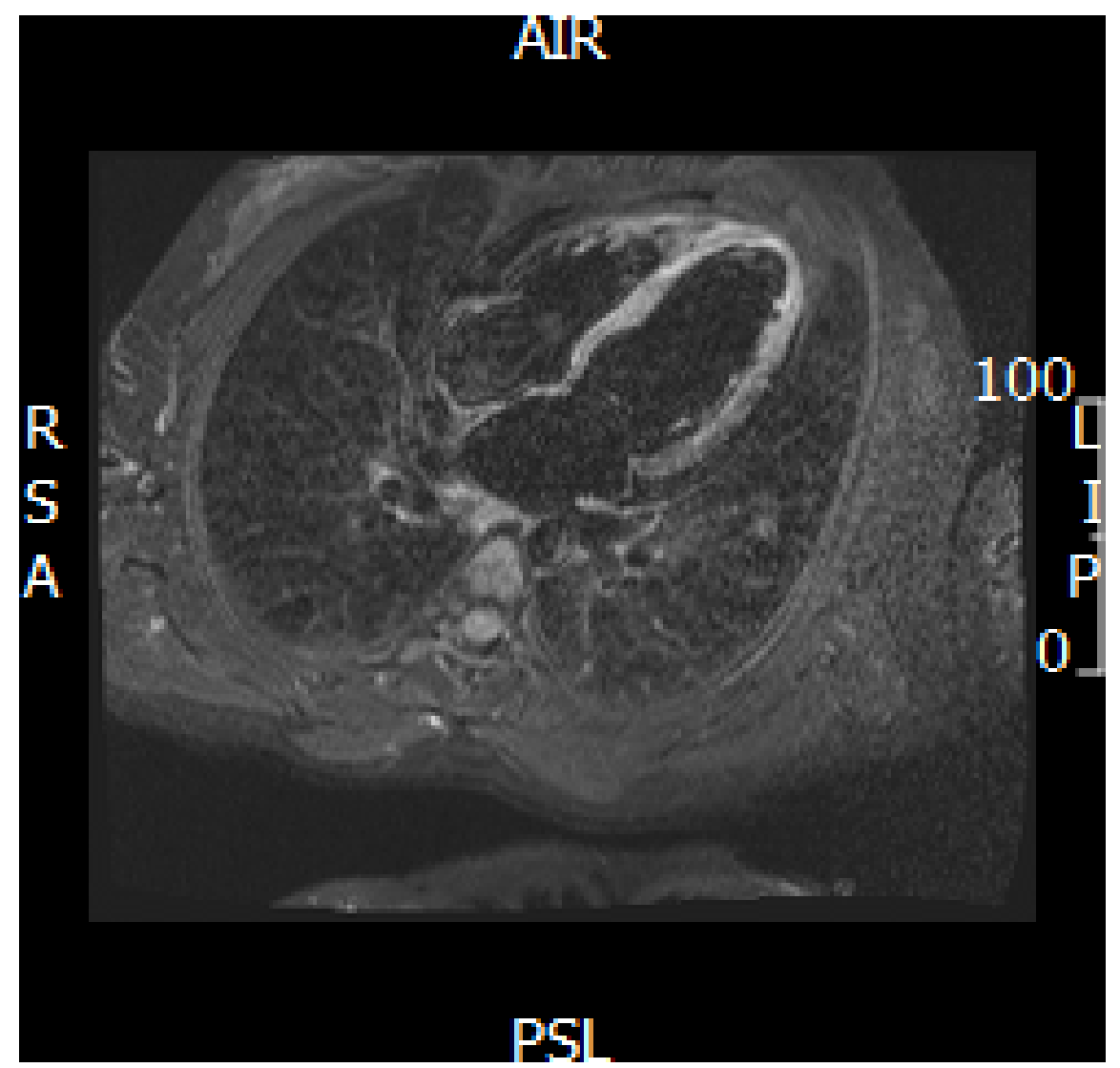




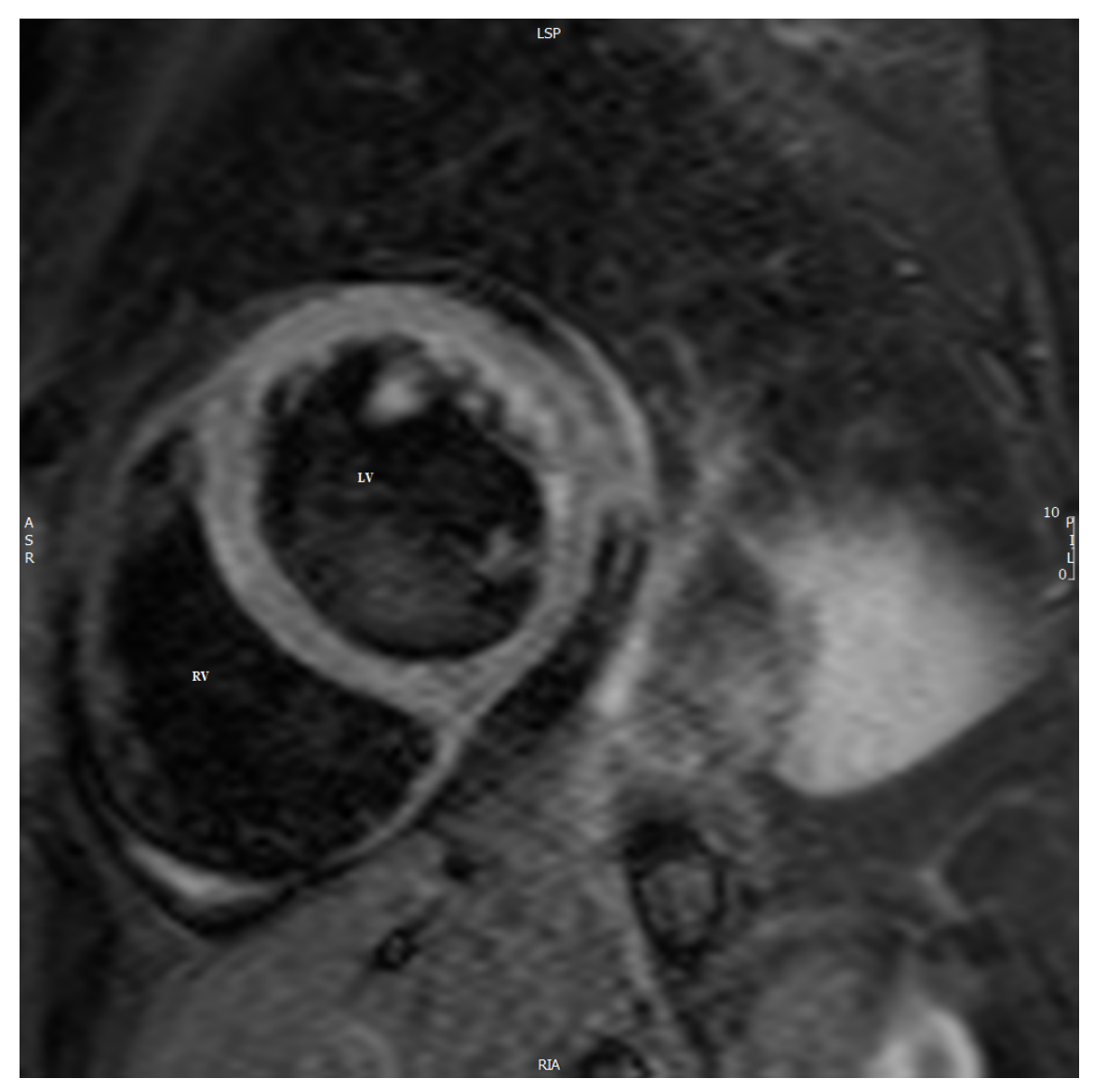




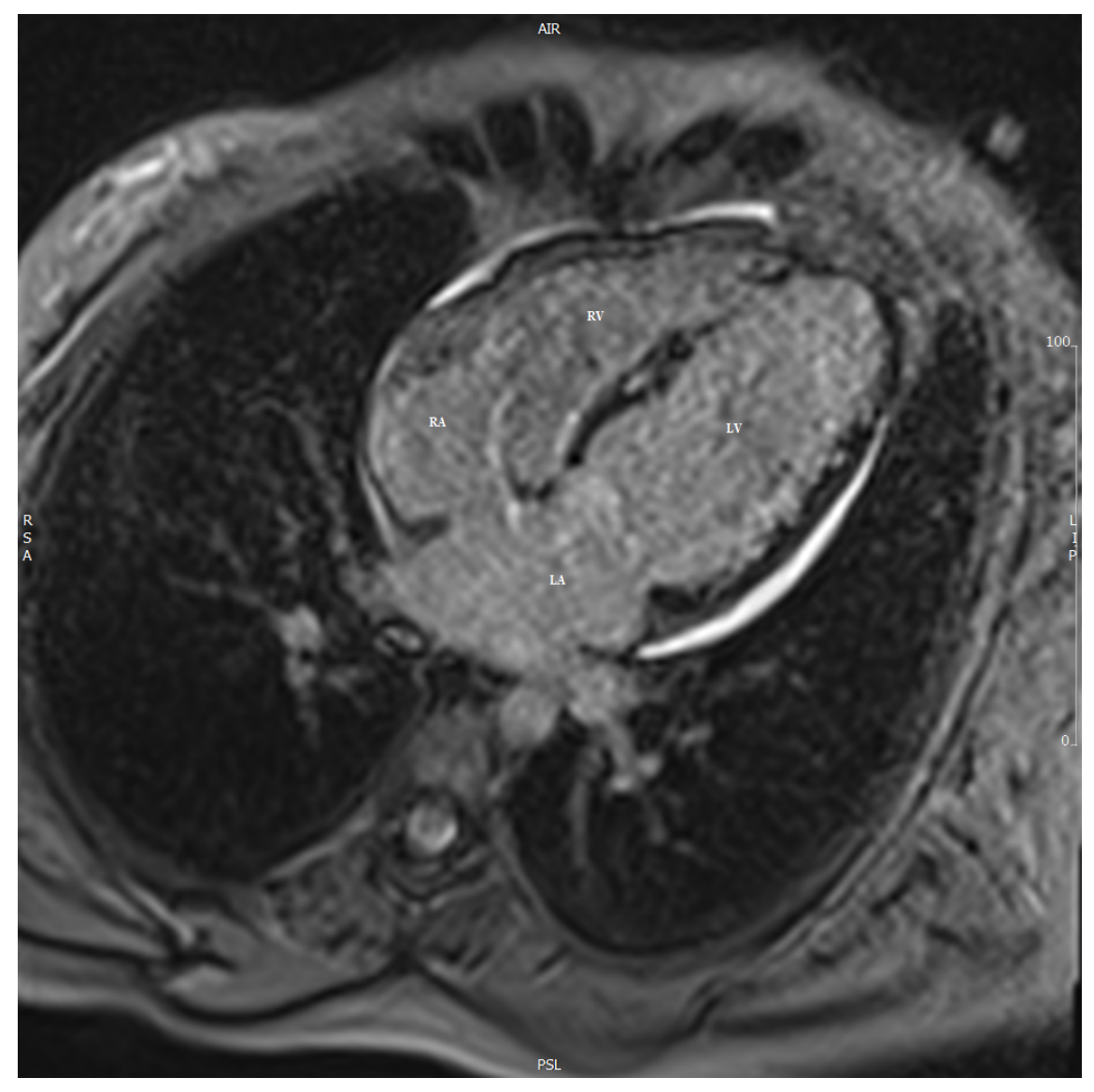



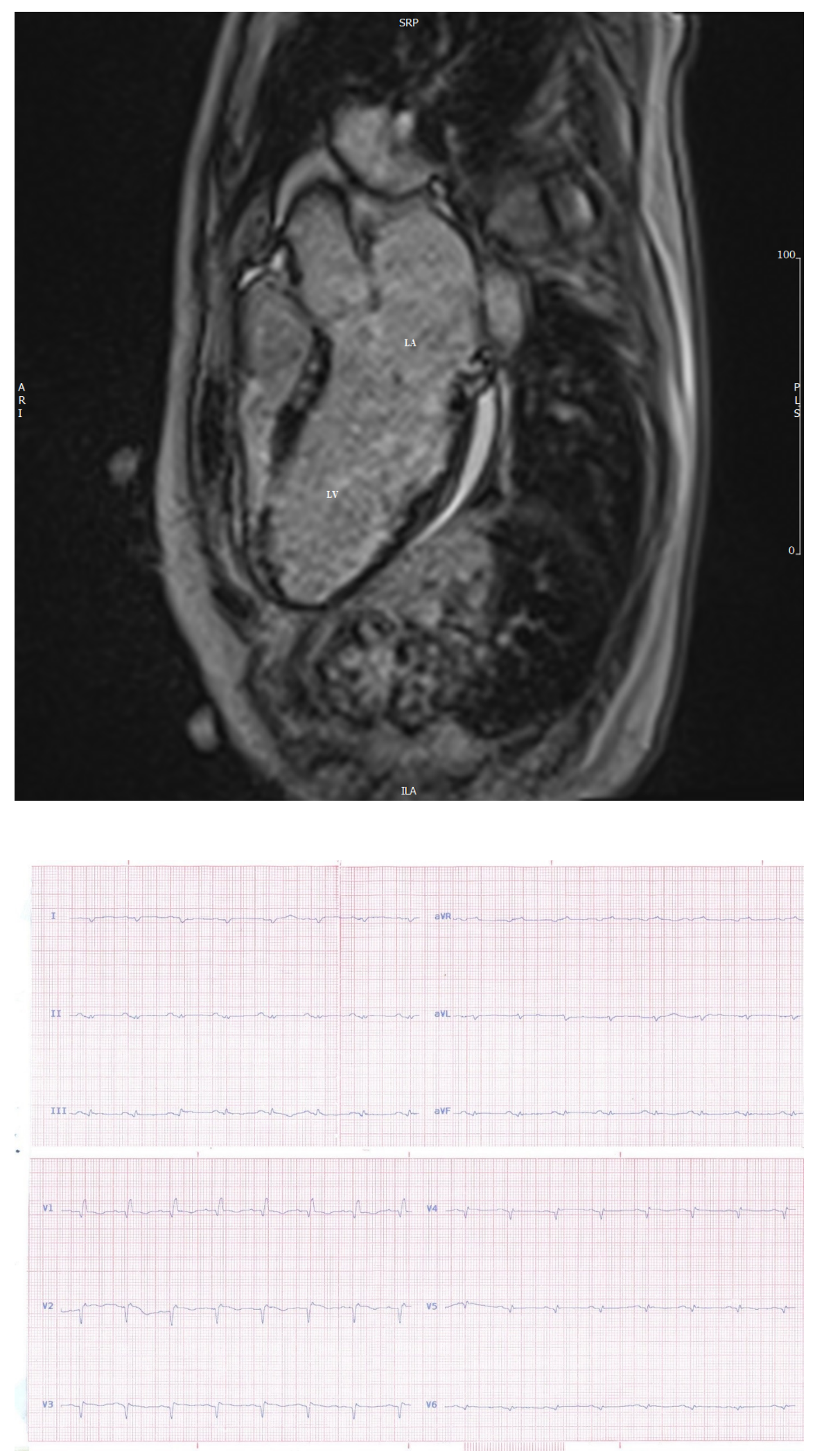

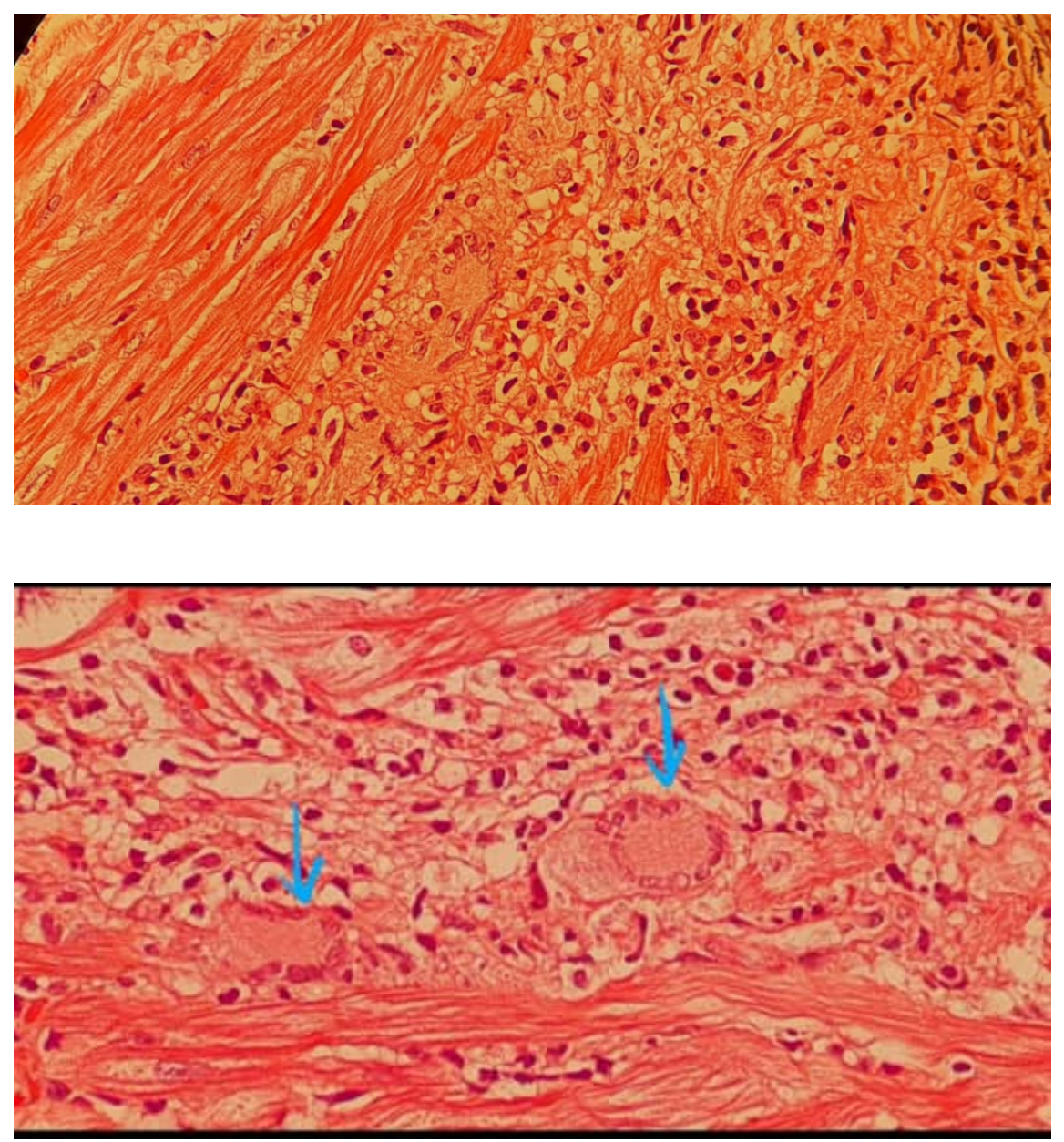